\title{
An expression for the Auger backscattering factor and the $\phi(0)$ function at oblique incidences
}

\author{
Jacques Cazaux
}

LASSI, GRSM, Faculté des Sciences, BP 347, 51062 Reims Cedex, France

(Received February 24, 1992; accepted May 13, 1992)

Résumé. - Basée sur des arguments physiques simples, l'expression proposée:

$$
\phi(0)-1=R=\frac{\eta}{(1+\eta)}[1+3 \cos \alpha]\left[1-U_{0}^{-1}\right]\left[1+\ln \frac{1+\eta}{2} \cdot \ln ^{-1} U_{0}\right]
$$

rend compte analytiquement de l'évolution du coefficient de rétrodiffusion Auger $r(=1+R)$ et de la fonction d'ionisation de l'EPMA à la surface $\phi(0)$, en fonction de l'angle d'incidence $\alpha$ des électrons. Elle conduit à une procédure inédite de quantification en AES et en EPMA, à savoir la mesure du coefficient de rétrodiffusion $\eta$ via la mesure du courant du porte échantillon correctement polarisé pour déterminer localement $r$ et $\phi(0)$ sans connaissance, $a$ priori de la composition du substrat. Son domaine d'application concerne donc l'analyse ponctuelle et l'acquisition d'images par spectroscopies Auger et d'émission $\mathrm{X}$ avec une mention particulière pour l'acquisition des profils en $z$ par AES et l'analyse des revêtements superficiels en EDS EPMA. Les prédictions déduites de l'expression proposée sont en excellent accord (au moins pour $U_{0} \geq 3$ ) avec les valeurs purement numériques déduites de simulations de Monte Carlo ou des solutions de l'équation de transport proposées par d'autres auteurs. Certaines incidences concernant l'influence de la rugosité de surface sur les mesures sont aussi évoquées.

Abstract. - Based on simple physical arguments, the proposed analytical expression:

$$
\phi(0)-1=R=\frac{\eta}{(1+\eta)}[1+3 \cos \alpha]\left[1-U_{\mathrm{o}}^{-1}\right]\left[1+\ln \frac{1+\eta}{2} \cdot \ln ^{-1} U_{0}\right]
$$

describes the evolution as a function of the incident angle $\alpha$, of the Auger backscattering factor $r(=1+R)$ and of the ionization function of EPMA at the surface, $\phi(0)$. This expression leads to a new procedure of quantification in AES and EPMA by the measurement of the electron backscattering coefficient $\eta$ via the measurement of electric current of the correctly polarized specimen holder, which allows the local determination of $r$ and $\phi(0)$ without the knowledge of the substrate composition. Its field of application concerns point analysis and images acquisition in Auger electron spectroscopy and $\mathrm{X}$-ray emission spectroscopy with a special attention for the in-depth profiling by AES and the non destructive profilometry of coatings by EDS-EPMA. The results deduced from the proposed expression are in excellent agreement (at least for $U_{\mathrm{o}} \geq 3$ ) with the numerical values deduced from Monte Carlo simulations and from the solutions of the transport equation as proposed by other authors. Some aspects related to the influence of the roughness of the surface on the measurements are also indicated. 


\section{Introduction.}

It is a well known fact that the Auger backscattering factor, $r=1+R$, describing the reinforcement of the Auger signal by the backscattered electrons, is one of the main parameters to be known for quantifying the surface composition of a solide by AES [1]. Nevertheless, despite efforts devoted to the evaluation of this matrix effect in various specific situations ([1 - 6] and references therein) there are only a few algebraic expressions able to give, in general, a numerical values of $r$. In fact the unique exception we know at oblique incidence are the expressions proposed by Shimizu and derived from Monte Carlo simulations [6] but these expressions only apply at selected angles of incidence $\alpha: \alpha=0^{\circ} ; 30^{\circ} ; 45^{\circ}$ and the results are expressed as a function of the subtrate atomic number, $Z$, not directly accessible to the Auger measurements.

On the other hand, in quantitative electron probe microanalysis, (E.P.M.A.) the quantification procedures often require the knowledge of the ionization function $\phi(\rho z)$ and its surface value, $\phi(0)$. Unfortunately althrough many empirical or semi empirical models of $\phi(0)$ have been established, ([7-10] references therein), all are devoted to the normal incidence case except the functional expression proposed by Pouchou et al. [11]. This last expression has been also derived from Monte Carlo simulations, it can be applied at any angle of incidence but it also requires the knowledge of the substrate atomic number.

According to Castaing [12], the $\phi(\rho z)$ function is defined by the ratio of the intensities emitted by the layer $\mathrm{d} z$ at various depths into the specimen and the intensities emitted under the same conditions by an identical layer $\mathrm{d} z$ isolated in space and submitted to the same normal (or oblique) electron bombardment. For a thin layer at the surface of the investigated specimen, this definition of $\phi(0)$ corresponds to that of the Auger backscattering factor, $r$, [13], and at oblique incidence the path length of each incoming electron through the thin layer isolated in space is $\mathrm{d} z / \cos \alpha$.

The goal of the present paper is to provide an analytical expression for $r($ or $\phi(0))$ as a function of the angle of incidence $\alpha$ and of the electron backscattering factor, $\eta$, that can be measured during the analysis. This approach, which does not require the knowledge of the substrate composition, is an extension of that recently proposed by the author for the normal incidence situation [14] which has led to a 8.4\% deviation when compared to 79 experimental values [15].

If, in the present paper $\eta$ is next expressed as a function of the substrate composition, this is to compare the validity of the proposed expression to other theoretical and experimental results (requiring this knowledge).

In the normal procedure, suggested here, the proposed expression will be associated with the measurement of $\eta$ during analysis by using an experimental arrangement similar to that used by Barkshire et al. for Auger [16] or better that used by Bishop for some electron backscattering measurements (specimen current, Fig. 2 in Ref. [17]). Such a procedure will be useful for automatic removal of substrate backscattering effects in Scanning Auger Microscopy and Auger depth profiling of heterogeneous substrates but also in EPMA analysis of bulk materials (for the $\phi(0)$ determination) or of thin coatings on substrates when performed at oblique incidence in a Scanning Electron Microscope (SEM) with an Energy Dispersive System (EDS).

Lastly, the roughness of a surface leads to a local change of the incident angle $\alpha$ and the proposed approach will allow to estimate the influence of this roughness on the quantification procedures of EPMA and Auger.

\section{The proposed expression.}

2.1 GENERAL AND NORMAL INCIDENCE CASE. - From their definitions, the fundamental expressions of the Auger backscattering correction factor [4], $r=1+R$, (where $R$ describes the 
reinforcement of the Auger signal by the backscattered electrons with respect to the direct production by the incident beam, 1) and the $\phi(\rho z)$ function at the surface are given by:

$$
\phi(0)-1=R=\frac{\cos \alpha}{Q\left(E_{\mathrm{o}}\right)} \int_{E_{\mathrm{c}}}^{E_{\mathrm{o}}} \int_{2 \pi} \frac{\partial^{2} \eta}{\partial E \partial \Omega} \frac{Q(E) \cdot \mathrm{d} \Omega}{\sin \theta} \cdot \mathrm{d} E
$$

where $Q$ is the ionization cross-section of the element of interest on the surface and $\eta$ is the backscattered fraction of the incident current. $\cos \alpha$ (outside the integral) and $\sin \theta$ (inside the integral) are related to the path lengthes of the incoming and of the backscattered electrons respectively $(\theta$, being measured with respect to the surface).

At normal incidence ( $\cos \alpha=1$ ), the expression proposed recently by the author [14] is deduced from equation (1), in which, the following assumptions simplify the result:

i) The spectral distribution of the backscattered electrons $\partial \eta / \partial E$ is replaced by a $\delta$-function located at:

$$
\bar{E}=(1+\eta) E_{\mathrm{o}} / 2,
$$

(following the approach initiated by Seiler [18]). The weight of this $\delta$ function is choosen to be $\eta_{\text {eff }}=\eta\left(1-1 / U_{\mathrm{o}}\right)$ to take into account only the efficient backscattered electrons, $E>E_{\mathrm{c}}$, where $U_{\mathrm{o}}$ is equal to $E_{\mathrm{o}} / E_{\mathrm{c}}$ and $E_{\mathrm{o}}$ is the primary beam energy.

ii) In agreement with the experimental results, the angular distribution of the backscattered electrons is choosen to follow a Lambert distribution of the form [19]:

$$
\frac{\partial \eta}{\partial \Omega}=\frac{\eta}{\pi} \cos \beta
$$

where $\beta$ is the emission angle of the backscattered electrons with respect to the normal.

Inserting equation (3) in equation (1) and integrating it over $2 \pi \operatorname{sterad}(\mathrm{d} \Omega=2 \pi \sin \beta$.d $\beta$ ), leads to the factor 2 appearing in the intermediate result:

$$
R(0)=2 \eta_{\mathrm{eff}} \cdot \frac{Q(\bar{E})}{Q\left(E_{\mathrm{o}}\right)}
$$

iii) Lastly a simplified Bethe cross-section of the form $Q(E) \propto \frac{1}{U} \ln U$ is used. Better expressions for the cross-sections can be used (such as that proposed by Gryzinski) but the improvement is not significant because the choice of a less accurate cross-section leads to errors that more or less cancel each other because they appear in the numerator and in the denominator of equation (1).

The result thus obtained is:

$$
R=\phi(0)-1=\frac{4 \eta}{1+\eta}\left[1-U_{\mathrm{o}}^{-1}\right]\left[1+\ln \left(\frac{1+\eta}{2}\right) \cdot \ln ^{-1} U_{\mathrm{o}}\right]
$$

For a comparison with published experimental results, the Hunger and Kuchler expression [20] for $\eta$ has been next choosen for expressing the result as a function of the substrate composition $Z$, in order to compare the numerical values of equation (5) to the experimental results. This expression is:

$$
\eta=E_{\mathrm{o}}^{m(Z)} . c(Z)
$$

with $m(Z)=0.1382-\frac{0.9211}{\sqrt{Z}}$ and $c(Z)=0.1904-0.2236 \ln Z+0.1292 \ln ^{2} Z-0.01491 \ln ^{3} Z$. 
One advantage of such an expression is the fact that it takes into account the (slight) change of the backscattering coefficient values $\eta$ when the primary energy $E_{\mathrm{o}}$ (expressed in keV in Eq. (6)) changes.

When the predictions thus deduced from equation (5) were compared to 79 experimental values concerning a wide variety of thin coatings $Z_{1}$ on substrates $Z_{2}$, the authors were surprised to find a standard deviation of $7-8 \%$ very close to the experimental precision [15]. In fact, it is believed that this success is certainly due to the fact that the comparison was restricted to rather high primary beam energies $E_{\mathrm{o}}(\geq 10 \mathrm{keV})$ and binding energies $E_{\mathrm{C}}$ below $2.5 \mathrm{keV}$, i.e. $U_{\mathrm{o}} \geq 4$. In these situations, most of the backscattared electrons have a kinetic energy in an energy range where the cross section is decreasing monotonically leading, to a cancellation of the errors induced by the crude assumption $\mathbf{i}$ (see Fig. 1 for an illustration).

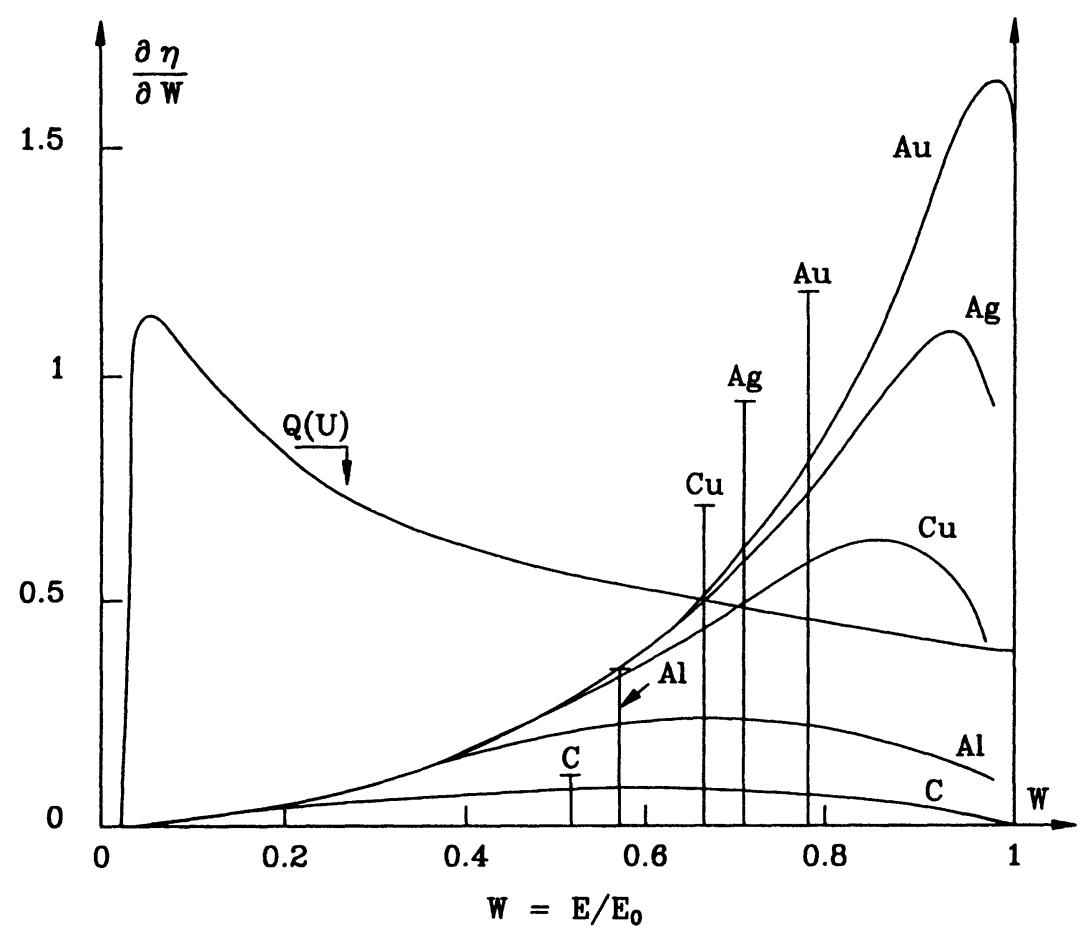

Fig. 1. - Energy distribution of backscattered electrons, $\frac{\partial \eta}{\partial W}$, for various elements as a function of the normalized energy $W=E / E_{\mathrm{o}}$ measured by Darlington [21], overlaid with the Bethe ionization cross-section, $Q(U)$ for a $1 \mathrm{keV}$ core energy and a $30 \mathrm{keV}$ primary beam. The simplification i consists in substituting a $\delta$ function (of weight $\eta_{\text {eff }}$ ) located at $E_{\mathrm{o}}\left(\frac{1+\eta}{2}\right)$ to the experimental distribution $\frac{\partial \eta}{\partial W}$.

2.2 OBLIQUE INCIDENCE CASE. - The success of equation (5), at normal incidence suggests the application of the same philosophy to the oblique situation and the new expression to find has to give the same result at the normal incidence limit $(\alpha=0)$. When $\alpha$ is increased, the physical changes concern the increase of the amplitude of $\eta$, the changes of its spectral and angular distribution, with also the influence of $\cos \alpha$ in equation (1). 
i) The change in amplitude of $\eta$ is taken into account by substituting $\eta(\alpha)$ (at oblique incidence) to $\eta(0)$ (at normal incidence) as a normalization factor.

ii) For the shift towards higher energy of the spectral distribution, $\frac{\partial \eta}{\partial E}$, observed when $\alpha$ is increased [21], it is postulated that this shift is correctly described by the corresponding shift of the mean energy:

$$
\bar{E}=\frac{E_{\mathrm{o}}}{2}(1+\eta(\alpha))
$$

iii) When $\alpha$ is increased (see Fig. 2) starting from a nearly cosine distribution, the backscattered intensity is progressively peaked around the angle of regular reflection.
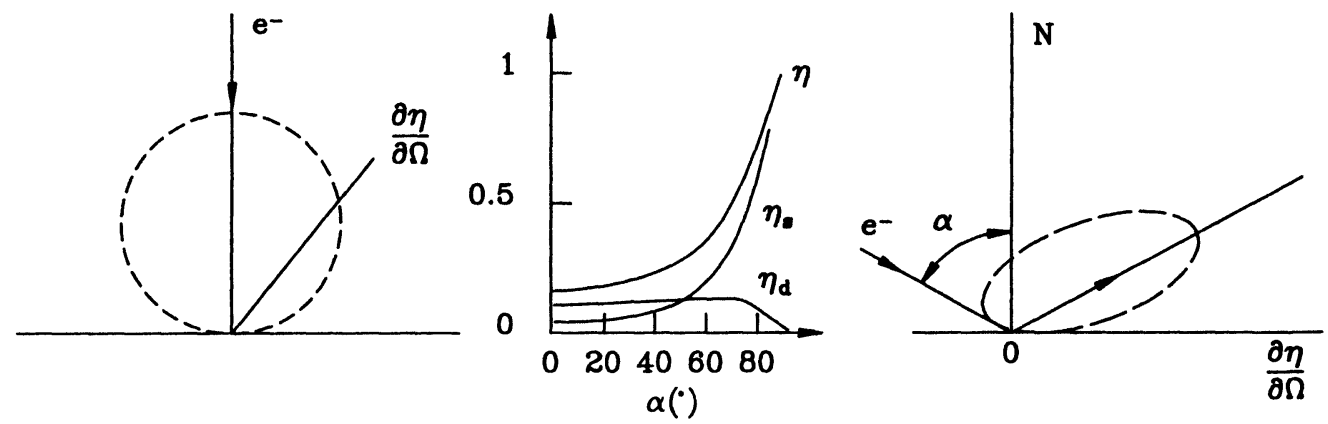

Fig. 2. - Experimental angular backscattering distribution for Al. Left: cosine distribution at normal incidence. Right: distribution at oblique incidence $\alpha=60^{\circ}$. In the middle, evolution of $\eta$ and of its two components $\left(\eta_{\mathrm{d}}\right.$ and $\left.\eta_{\mathrm{S}}\right)$ as a function of $\alpha$. All the three curves are taken from Niedrig's paper [22].

Following Niedrig [22], $\eta(\alpha)$ has two components, the single scattering fraction $\eta_{\mathbf{S}}$ and the diffuse scattering fraction $\eta_{\mathrm{d}}$. This last fraction has a quite constant weight in the interval $0<\alpha<$ $60^{\circ}-70^{\circ}$. Its angular distribution follows a Lambert law (as it can be shown from the Archard's model) and its contribution to $R$ is that of the normal incidence case weighted by $\cos \alpha$ (related to the path length of incoming electrons): $2 \eta_{\mathrm{d}} \cos \alpha$.

To describe the angular contribution of $\eta_{\mathrm{S}}$, one has to find a function of $\alpha$ starting from 2 (associated to the Lambert distribution of this fraction at $\alpha=0^{\circ}$ ) and going progressively to unity as $\alpha$ increases towards the grazing incidence. This limit (unity) is justified by the fact that at large incident angles the single scattering fraction is responsible of the peak shape of $\eta$ around the angle of regular reflection and the effects of such specularly reflected electrons are the same as that of the incoming electrons. One of the simplest function satisfying these boundary conditions is $(1+$ $\cos \alpha$ ) and we select it.

We also suppose that the relative weight of these two contributions is the same. In fact the equality of the two only occurs at around $\alpha=40^{\circ}-60^{\circ}$ (see Fig. 24 in Ref. [22] and, here, Fig. 2, in the middle), but for lower $\alpha$ angles the choice of the weight is unimportant because the two contributions are of a Lambert type. The result of the above analysis can be written in the form:

$$
\frac{2 \eta(\alpha)}{2} \cos \alpha+\frac{\eta(\alpha)}{2}(1+\cos \alpha)
$$


Consequently, the proposed expression for $R(\alpha)$ is:

$$
R(\alpha)=\frac{\eta(\alpha)}{2}(3 \cos \alpha+1)\left[1-\frac{1}{U_{\mathrm{o}}}\right]\left[\frac{Q(\bar{E})}{Q\left(E_{\mathrm{o}}\right)}\right]
$$

where $\bar{E}=\frac{E_{\mathrm{o}}}{2}[1+\eta(\alpha)]$.

Using again a simplified Bethe cross-section, this expression became:

$$
R(\alpha)=\frac{\eta}{(1+\eta)}(3 \cos \alpha+1)\left[1-U_{\mathrm{o}}^{-1}\right]\left[1+\ln \frac{1+\eta}{2} \cdot \ln ^{-1} U_{\mathrm{o}}\right]
$$

in which $\eta$ means $\eta(\alpha)$-the symbol $\alpha$ will be next omitted for the sake of the simplicity-.

\section{Results.}

3.1 ReSUltS AT $10 \mathrm{keV}$ FOR $\mathrm{Si}, \mathrm{Cu}, \mathrm{Ag}, \mathrm{W}$. - The predictions of equation (8) have been evaluated for four elements $(Z=14 ; 29 ; 47 ; 74)$ at a primary beam energy of $10 \mathrm{keV}$. For the first three elements, the experimental values of $\eta(\alpha)$ measured by Dresher have been used [23] (results reported by Kanaya and Ono for Si and Cu, Fig. 6 in Ref. [24] and by Niedrig, Fig. 24 in Ref. [22]). For tungsten, because of the lack of experimental data, we have evaluated it, using equation (9) derived from the work of Arnal et al. [25]:

$$
\eta(\alpha)=\eta(0)\left(\frac{2}{1+\cos \alpha}\right)^{p}
$$

with $p=-\ln \eta(0) / \ln 2 \quad$ and $\quad \eta(0)=0.483$ (Bishop [17]).

The values thus obtained are shown (full symbols) in figures $3 \mathrm{a}$ to $3 \mathrm{~d}$. They have been compared to the results of transport calculations performed by Batchelor $e t$ al. [4] and to the functional expressions proposed by Shimizu (and derived from Monte Carlo simulations) [6]. These expressions are reproduced here as equations (10):

$$
\begin{aligned}
& \alpha=0^{\circ}, r=1+\left(2.34-2.1 Z^{0.14}\right) U^{-0.35}+\left(2.58 Z^{0.14}-2.98\right) \\
& \alpha=30^{\circ}, r=1+\left(0.462-0.777 Z^{0.2}\right) U^{-0.32}+\left(1.15 Z^{0.2}-1.05\right) \\
& \alpha=45^{\circ}, r=1+\left(1.21-1.39 Z^{0.13}\right) U^{-0.33}+\left(1.924 Z^{0.13}-1.88\right)
\end{aligned}
$$

The comparison with the functional expressions (also derived from M.C. simulations) proposed by Pouchou et al. is also shown [11]; these last expressions are:

$$
\phi_{\alpha}(0)=q\left(\frac{\phi_{\mathrm{o}}(0)}{q}\right)^{p}
$$

with

$$
\begin{aligned}
& p=(\cos \alpha)^{0.7} ; q=1+h\left[1-\exp \left(-U_{\mathrm{o}}-1\right)^{0.3}\right] ; \\
& h=0.2+2.3 Z^{-1 / 2} ; \phi_{\mathrm{o}}(0)=1+3.3\left(1-U^{k}\right) \eta^{1.2} \\
& k=-2+2.3 \eta \text { and } \eta=1.75 \times 10^{-3} Z+0.37\left(1-\mathrm{e}^{-0.015 Z^{1.3}}\right)
\end{aligned}
$$



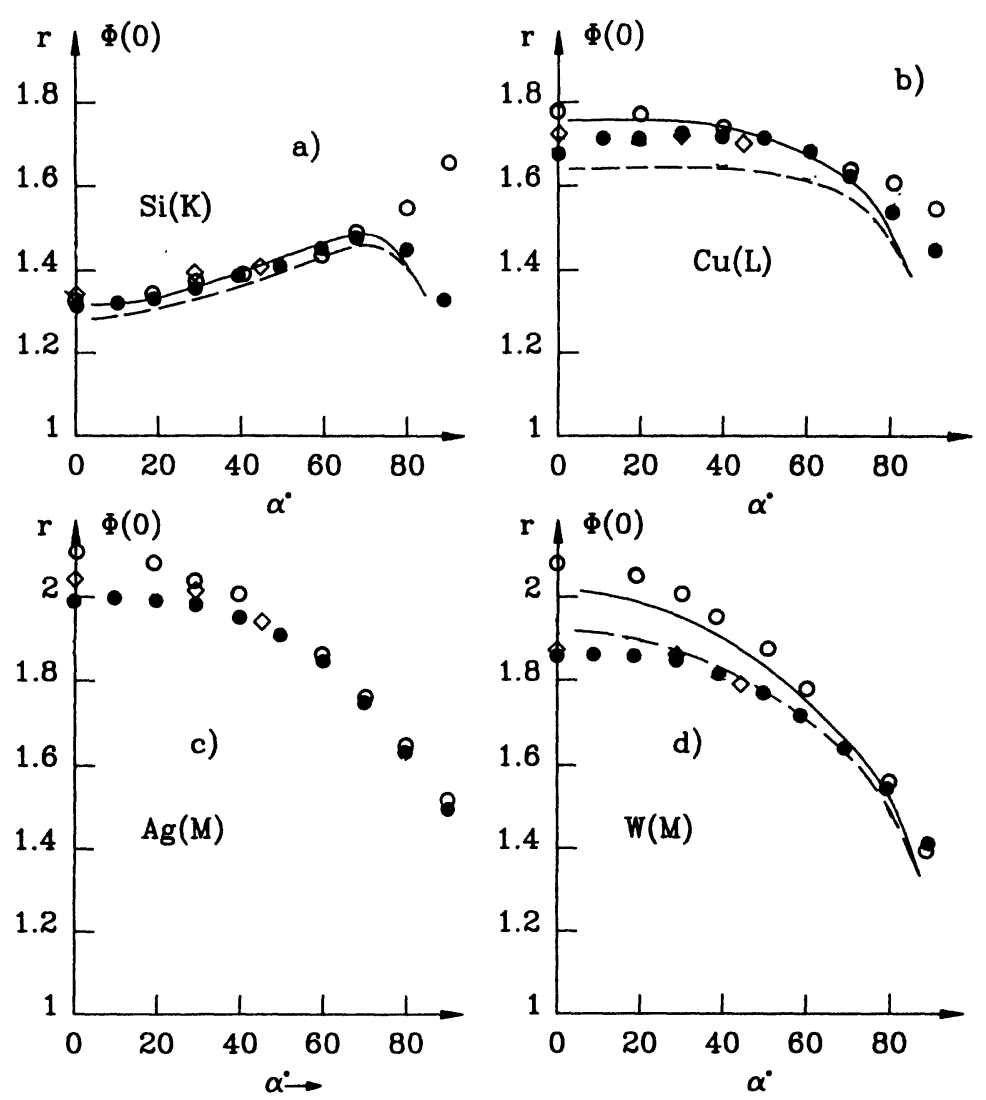

Fig. 3. - Auger backscattering factor as a function of the angle of incidence for: a) $\mathrm{Si}(\mathrm{K})$; b) $\mathrm{Cu}(\mathrm{L})$; c) $\mathrm{Ag}(\mathrm{M})$ and d) $W(\mathrm{M})$ at $E_{\mathrm{o}}=10 \mathrm{keV}:(\bullet)$ full symbols correspond to the proposed approach (Eq. (8)) using experimental values of $\eta(\alpha)$ except for $W$ where they are calculated (see text); ( $\diamond)$ Open lozenges and open circles (o) correspond to results deduced from M.C. simulations: equations (10) [5] and (11) [11] respect. The curves correspond to solutions of the transport equation using Hartree Fock cross-section (full line) and Lotz cross-section (broken line) [4].

As also shown in table I, the agreement between the proposed expression and all the other approaches is, as a whole, excellent. For $\mathrm{Si}$, when $\alpha$ is increased the proposed expression describes very well the behaviour deduced from transport calculations [4]: first, the increase of $r$, the fact that it passes through a maximum after which it rapidly falls. For this element all the methods lead to values close to each other to less than few percent in the angular range $0-70^{\circ}$.

The unique deviation concerns the Pouchou et al. expression which leads to a continuous increase instead of decrease but only for $\alpha>70^{\circ}$.

For the other three heavier elements, all the approaches describe the fact that $r$ is first a constant and then decreases and the general trends are well described by equation (8). For $\mathrm{Ag}$, a significant difference is observed between the absolute values of $r$ obtained from Pouchou's equation at normal and others but the difference rapidly vanishes when $\alpha$ is increased. The same situation holds for $\mathrm{W}$ here the transport method gives values between that deduced from Pouchou's expression on the one hand, and that deduced from the proposed approach as well as that of Shimizu on the other hand. In the case of W, we believe the transport method is too optimistic 
Table I. - Comparison between the calculated values of $r$ (Auger) - or $\phi(0)$ (EPMA) using equation (8) of the present paper $C$ (p.p.) and equation (10) (from Shimizu [6]) and equation (11) (from Pouchou [11]) (at $\left.E_{\mathrm{o}}=10 \mathrm{keV}\right)$.

\begin{tabular}{|c|c|c|c|c|c|c|c|c|}
\hline \multicolumn{5}{|c|}{$\mathrm{Si}(\mathrm{K})$} & \multicolumn{4}{|c|}{$\mathrm{Cu}(\mathrm{L})$} \\
\hline$\alpha$ & $\eta$ & $\begin{array}{c}\text { Eq. (8) } \\
\text { C (p.p.) }\end{array}$ & $\begin{array}{c}\text { Eq. (10) } \\
\text { S[6] }\end{array}$ & $\begin{array}{c}\text { Eq. (11) } \\
\text { P[11] }\end{array}$ & $\eta$ & $\begin{array}{c}\text { Eq. (8) } \\
\text { C (p.p.) }\end{array}$ & $\begin{array}{c}\text { Eq. (10) } \\
\text { S[6] }\end{array}$ & $\begin{array}{c}\text { Eq. (11) } \\
\text { P[11] }\end{array}$ \\
\hline 0 & 0.163 & 1.31 & 1.36 & 1.35 & 0.295 & 1.67 & 1.71 & 1.77 \\
\hline $30^{\circ}$ & 0.21 & 1.36 & 1.40 & 1.37 & 0.36 & 1.72 & 1.71 & 1.74 \\
\hline $45^{\circ}$ & 0.28 & 1.41 & 1.40 & 1.41 & 0.425 & 1.71 & 1.67 & 1.72 \\
\hline & & $\begin{array}{l}=1.84 \mathrm{keV} \\
=5.435\end{array}$ & & & & $\begin{array}{l}c=0.933 \\
o=10.71\end{array}$ & & \\
\hline
\end{tabular}

\begin{tabular}{|c|c|c|c|c|c|c|c|c|}
\hline \multicolumn{5}{|c|}{$\operatorname{Ag}(\mathrm{M})$} & \multicolumn{4}{|c|}{$\mathrm{W}(\mathrm{M})$} \\
\hline$\alpha$ & $\eta$ & $\begin{array}{c}\text { Eq. (8) } \\
\text { C (p.p.) }\end{array}$ & $\begin{array}{c}\text { Eq. (10) } \\
\text { S[6] }\end{array}$ & $\begin{array}{c}\text { Eq. (11) } \\
P[11]\end{array}$ & $\eta$ & $\begin{array}{c}\text { Eq. (8) } \\
\text { C (p.p.) }\end{array}$ & $\begin{array}{c}\text { Eq. (10) } \\
\text { S[6] }\end{array}$ & $\begin{array}{c}\text { Eq. (11) } \\
P[11]\end{array}$ \\
\hline 0 & 0.406 & 1.99 & 2.04 & 2.1 & 0.483 & 1.88 & 1.91 & 2.09 \\
\hline $30^{\circ}$ & 0.453 & 1.975 & 2.01 & 2.04 & 0.52 & 1.845 & 1.87 & 2.01 \\
\hline $45^{\circ}$ & 0.51 & 1.92 & 1.93 & 1.96 & 0.58 & 1.80 & 1.79 & 1.91 \\
\hline & & $\begin{array}{l}=0.37 \mathrm{ke} \\
=27\end{array}$ & & & & $\begin{array}{l}E_{c}=1.809 \\
J_{0}=5.527\end{array}$ & & \\
\hline
\end{tabular}

because it also leads to a backscattering coefficient of $\mathrm{W}(\eta(0) \simeq 0.53)[26]$ larger that of gold $(\eta(0) \simeq 0.5)[17]$ despite the lower atomic number $(\mathrm{W}=74 ; \mathrm{Au}=79)$.

The physical explanation of the behaviour of $r($ or $\phi(0))$ as a function of $\alpha$ can be given in terms of a competition between the increase of $\eta_{\alpha}$ and the decrease of the weighting parameter $\cos \alpha$ (in Eq. (1)). For light elements, starting from very low values of $\eta(\eta \leq 0.2)$, the increase of $\eta$ (towards the unity for $\alpha=90^{\circ}$ ) is, at first, greater than the decrease of the weighting parameter, $\cos \alpha$, (when $\alpha \leq 60^{\circ}$ ). The reverse occurs for heavier elements for which the starting value of $\eta$ is higher $(\eta \geq 0.3)$ and its increase being less rapid is overcompensated by the decrease of $\cos \alpha$. This description has to be modulated at large incident angles by the peak shape of $\eta$ around the regular angle of reflection - leading to unity in the first term into brackets in equations (7) or (8) - but all the other effects are only second order effects. This physical description explains the surprising success of the proposed approach.

3.2 OTHER RESULTS. - The other interesting aspects concern the study of the influence on $r$ or $\phi(0)$ - of the primary beam energy $E_{\mathrm{o}}$ and of the ionization energy $E_{\mathrm{c}}$ of the element of interest on the surface. 
As opposed to the Shimizu and Pouchou approaches where these two parameters act in a similar way only on the $U_{\mathrm{o}}$ values, it should be pointed out that the proposed approach takes into account the influence of $E_{\mathrm{o}}$ on $U_{\mathrm{o}}$ but also on $\eta$ while $E_{\mathrm{c}}$ only acts on $U_{\mathrm{o}}$.

To compare the proposed approach to other theoretical and experimental results, the difficulty associated to the lack of experimental results on $\eta(\alpha)$ at energies different from $10 \mathrm{keV}$ has been overcome in the following way:

i) $\eta\left(0^{\circ}, E_{\mathrm{o}}\right)$ at the energy $E_{\mathrm{o}}$ and at normal incidence is deduced from the experimental values of Dresher at $10 \mathrm{keV} \eta\left(0^{\circ}, 10 \mathrm{keV}\right)$ by using the power law of Hunger and Kuchler (Eq. (6)): $\eta\left(0^{\circ}, E_{\mathrm{o}}\right) / \eta\left(0^{\circ}, 10 \mathrm{keV}\right)=\left(E_{\mathrm{o}} / 10\right)^{m(Z)}$ with $E_{\mathrm{o}}$ in $\mathrm{keV}$.

ii) $\eta\left(\alpha^{\circ}, E_{\mathrm{o}}\right)$ is deduced from the Dresher's results at the same angle of incidence (and at $10 \mathrm{keV}), \eta\left(\alpha^{\circ}, 10 \mathrm{kV}\right)$ using:

$$
\begin{aligned}
\Delta \eta\left(\alpha^{\circ}\right) & =\eta\left(\alpha^{\circ}, E_{\mathrm{o}}\right)-\eta\left(\alpha^{\circ}, 10 \mathrm{keV}\right) \\
& =\Delta \eta\left(0^{\circ}\right) \cdot \cos \alpha
\end{aligned}
$$

Expression (12) is based on the fact that the backscattering factor approaches unity at any primary beam energy when $\alpha$ approaches $90^{\circ}$.

This procedure is intended to give (for $\eta$ ) calculated values closer to reality than the use of an expression in the form of equation (9). Another possibility (not explored) is to use the following expression [11]:

$$
\eta_{\alpha}=\eta_{\mathrm{o}}^{s} \text { with } s=(\cos \alpha)^{u}
$$

and $u=1.14-0.4(1-\exp -Z / 25)$.

The calculation of $r$ has been next performed (using Eq. (8)) for Si at 5 and $20 \mathrm{keV}$ primary beam energies. The results are shown in figure 4 where they are compared to Monte Carlo calculations and Transport Equation results of other authors (Jablonski [25], El Gomati et al. [3], Pouchou et al. [11] for MC, Batchelor et al. [4] for T. Eq.). It can be observed that the general shape of $R(\alpha)$ deduced from the proposed model is in agreement with the Batchelor's results. As at $10 \mathrm{keV}$, a slow increase at low incident angles is next followed by a maximum (in the $60^{\circ}-80^{\circ}$ range) and a rapid decrease (not described by Pouchou exp.).

A detailed comparison shows that the predictions of equation (8) are in good agreement with the MC calculation of Jablonski and the solutions of Transport Equation when a Powell crosssection is used for $E_{\mathrm{o}} \simeq 5 \mathrm{keV}$ and $0<\alpha<50^{\circ}$. In the same angular range, the results of Shimizu are slightly higher like the solutions of transport equation using the Hartree Fock crosssection or more the results of Pouchou. At higher angles of incidence $\left(\alpha>50^{\circ}\right)$ the proposed approach seems to give values slightly too low.

At $E_{\mathrm{o}}=20 \mathrm{keV}$, the proposed approach leads to results between those of Shimizu [6] and those deduced from the transport method [4] or from Pouchou expression.

If the proposed approach can be suspected for the results obtained at $5 \mathrm{keV}$ and oblique incidence $\left(\alpha>50^{\circ}\right)$ because of the very low reduced energy $(U \simeq 2.7)$ corresponding to such a situation, in the other cases, it is difficult to decide where are the best results.

The same remarks hold when the various calculations of $r$ as a function of $E_{\mathrm{o}}$ are performed for the silicon $\mathrm{K}$ line (Fig. 5a) and the $\mathrm{Cu} \mathrm{L}$ line (Fig. 5b).

For example, for the silicon case, we have seen that the proposed approach leads to values close to those of Jablonski and below those deduced from the transport method at $5 \mathrm{keV}$, while, at higher $E_{\mathrm{o}}$ energies, the proposed approach and MC calculations (Shimizu and Pouchou) are in excellent agreement (see Si at $\alpha=0^{\circ}$ ) and lead to values slightly higher than that of the transport method. Despite these slight differences the main point is that the proposed expression describes correctly the asymptotic behaviour of $r($ or $\phi(0))$ at beam energies greater than $10-15 \mathrm{keV}$. 


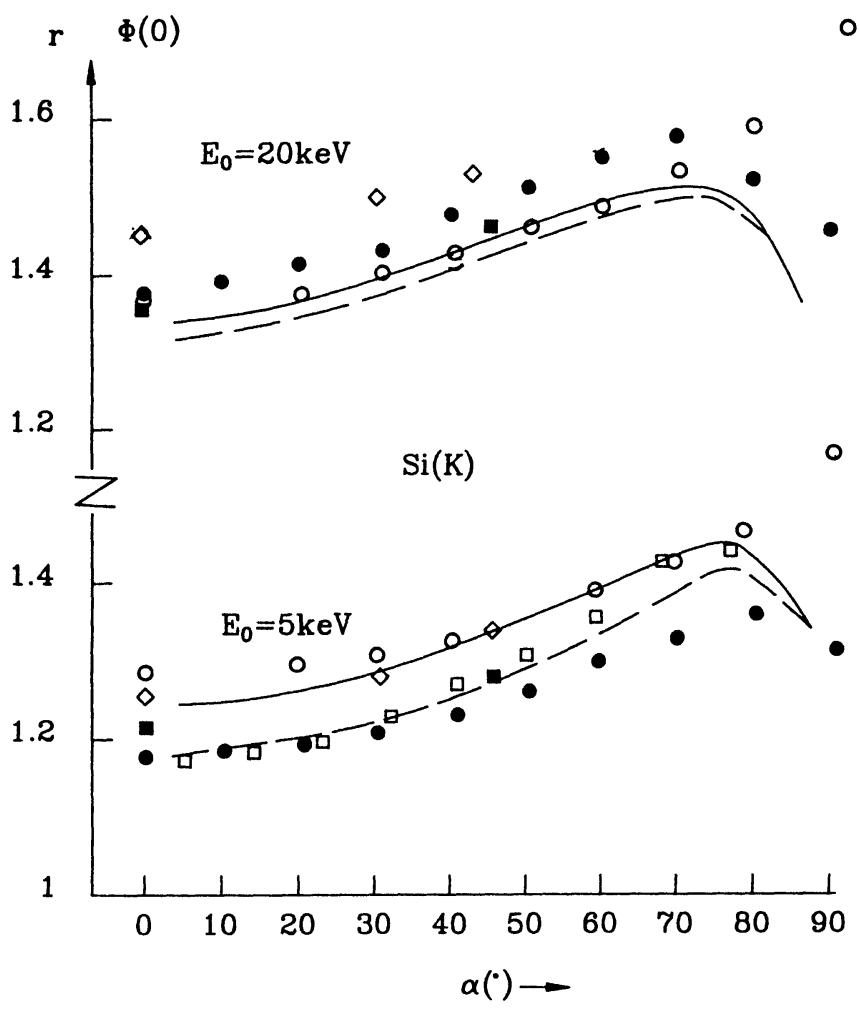

Fig. 4. - Auger backscattering factor as a function of the angle of incidence for $\operatorname{Si}(\mathrm{K})$ at $E_{\mathrm{o}}=5 \mathrm{keV}$ (bottom) and $E_{\mathrm{o}}=20 \mathrm{keV}$ (top). Full symbols $(\bullet)$ correspond to the proposed approach. Full squares, open squares, open lozenges correspond to M.C. simulations of El Gomati et al. [3], Jablonski [27] Shimizu [6] respect - as reported by Batchelor et al [4]. Open circles correspond to MC simulations of Pouchou et al. [11]. The lines correspond to transport equation results of Batchelor et al. [4] using two different cross-sections (full line: Hartree Fock; broken line: Powell).

Another interesting study is the influence (on $r$ ) of the ionization energy, $E_{\mathbf{c}}$, of analyzed element on the surface for different substrate compositions.

In the proposed approach, this influence is described by the overvoltage $\left(U_{\mathrm{o}}=E_{\mathrm{o}} / E_{\mathrm{c}}\right)$ and the primary beam energy has to be defined (for the choice of $\eta$ ). In figure 6 , we have choosen $E_{\mathrm{o}}=10 \mathrm{keV}$ but the results obtained on $\mathrm{Ag}$ substrates are independent of this choise because $\eta(\mathrm{Ag})$ is nearly a constant in a wide primary beam energy range $(5-40 \mathrm{keV})$ [18]. The results deduced from Shimizu's expression (and deduced from simulations at 3,5 and $10 \mathrm{keV}$ ) and from Pouchou's expression are also shown.

In figure 6 other published results have been also reported to obtain data in a rather wide range for $U_{\mathrm{o}}$ despite the fact they have been obtained at primary beam energies generally greater than $10 \mathrm{keV}$.

This is the case for Batchelor's results $(\mathrm{Si}(\mathrm{K}) / \mathrm{Si}$ at $20 \mathrm{keV})$ as well as the experimental results taken from a recent compilation [15] and from the thesis of Jbara [28]: $\mathrm{Cu}(\mathrm{M}) / \mathrm{Ag}$ and $\mathrm{Al}(\mathrm{K}) / \mathrm{Ag}$ at $15,20,25,30 \mathrm{keV}$ but also $\mathrm{Cu}(\mathrm{M}) / \mathrm{Si}$.

Here again, despite slight differences between the Shimizu approach, the Pouchou approach and the proposed approach (the first one leading systematically to values slightly higher than the 

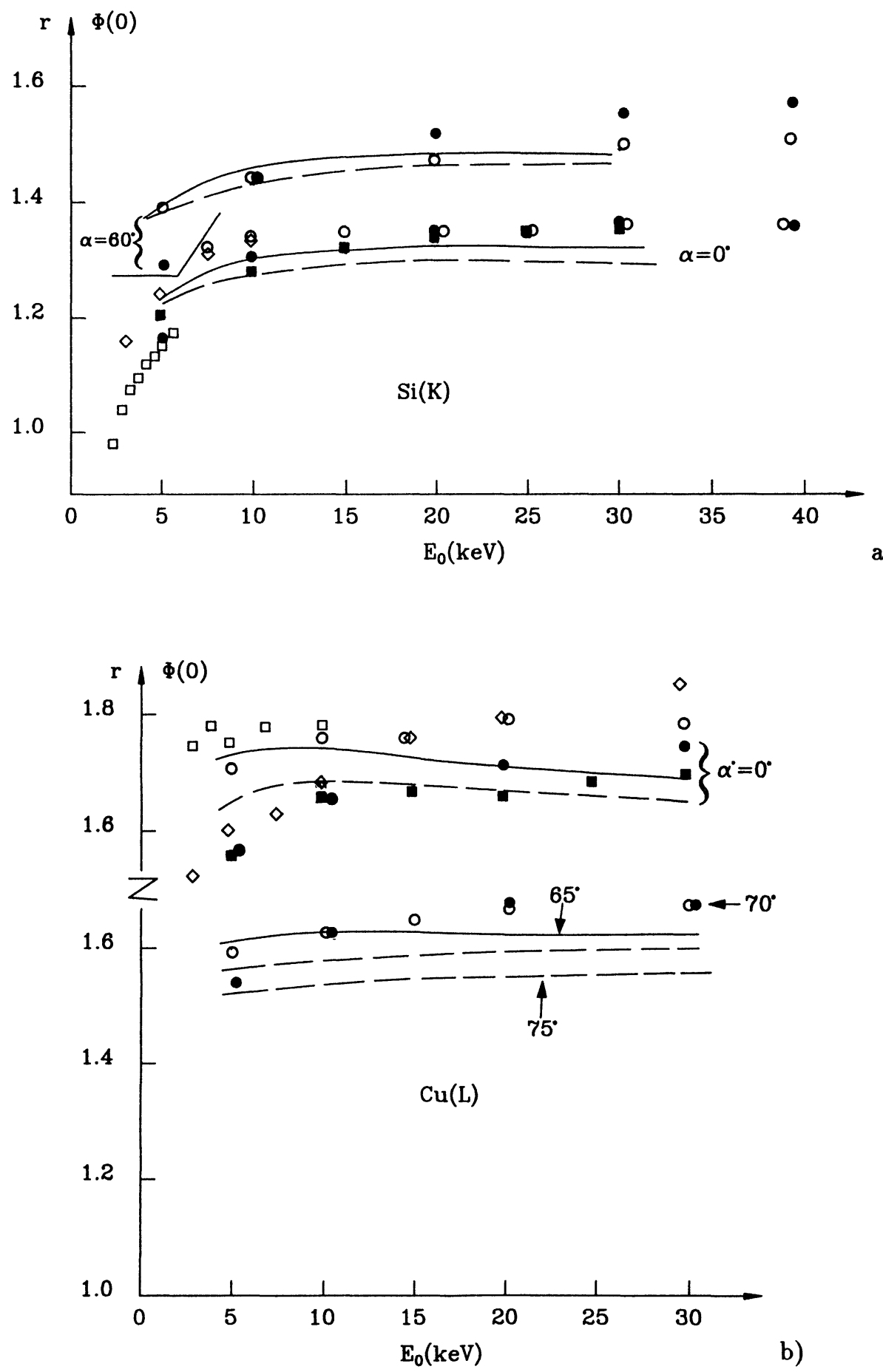

Fig. 5. - Auger backscattering correction factor - and $\phi(0)$ function - as a function of the probe energy for the ionizations of the $\mathrm{Si}(\mathrm{K})$ level (a) and of the $\mathrm{Cu}(\mathrm{L})$ level (b), and incident angles of $\alpha=0^{\circ}$ and $\alpha^{\circ}=60^{\circ}$ (Si case); $\alpha=0^{\circ}$ and $\alpha=70^{\circ}$ (Cu case). The symbols are the same as for figure 4 . 
third one at normal incidence), the general behaviour is the same with an asymptotic behaviour at reduced energies $U_{\mathrm{o}}$ greater than 10 (this asymptotic behaviour being pronounced in the Pouchou approach than that given by Shimizu's Eqs.).

Another remark on this behaviour concerns the fact that the difference between the three approaches is smaller at $\alpha=45^{\circ}$ than in the normal incidence situation. At $\alpha=45^{\circ}$ and for $\mathrm{Ag}$, the difference falls below the precision of the experimental results $( \pm 7 \%$ ) (see Fig. $6 \mathrm{~b})$.

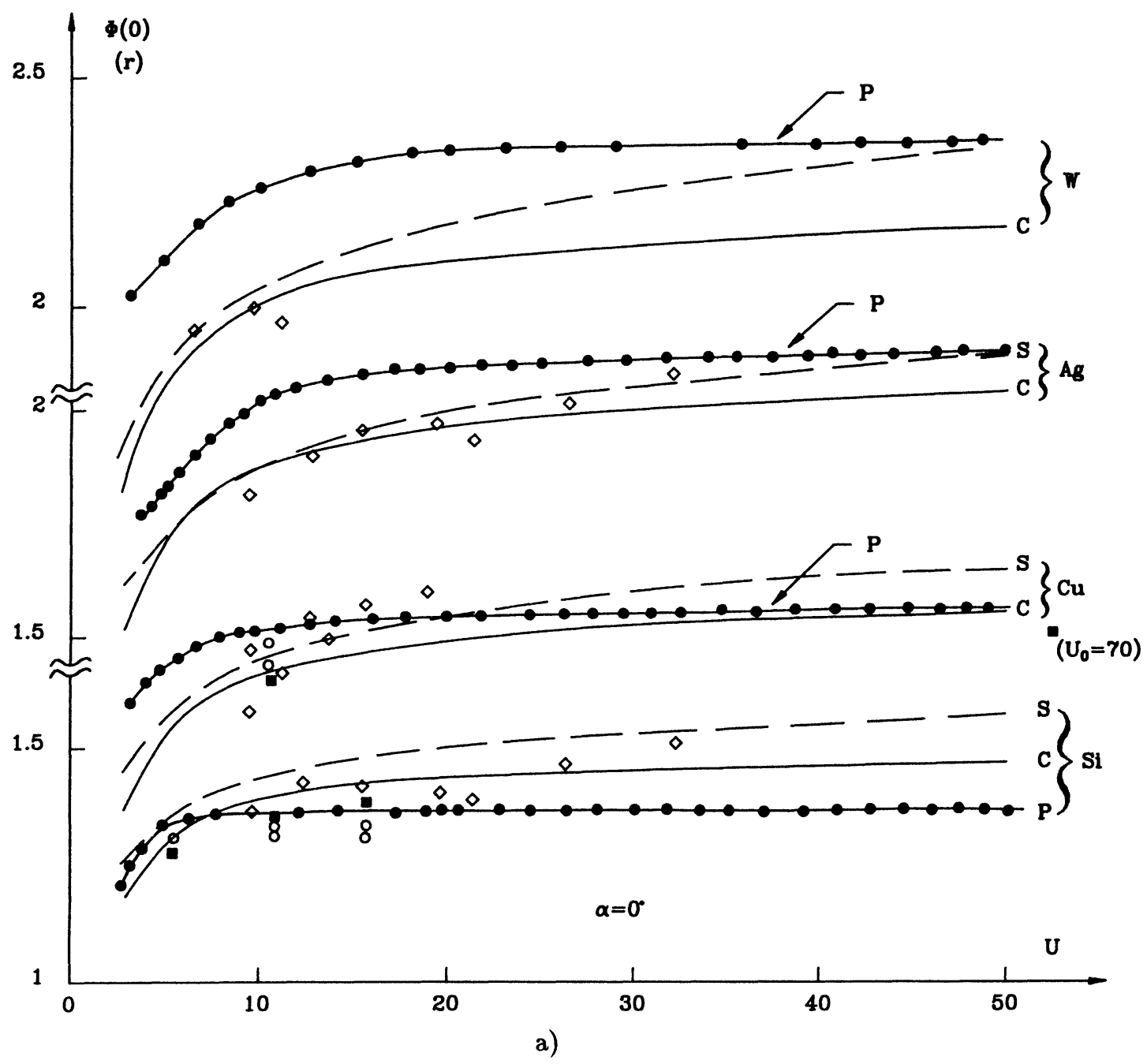

Fig. 6. - Auger backscattering correction factor - and $\phi(0)$ function - as a function of the reduced energy $(U)$ for four substrates $\mathrm{Si}, \mathrm{Cu}, \mathrm{Ag}$ and $\mathrm{W}$ and two different incident angles $\alpha=0^{\circ}$ (a) and $\alpha=45^{\circ}$ (b). The symbols are: full line: proposed expression - using $\eta(10 \mathrm{keV})$ as in figure 3 - (indicated C); broken line: Shimizu expressions [6] (indicated S), dotted lines: Pouchou expression [11] (indicated P); open circles: solutions of transport equation [4], full squares: El Gomati (M.C.) results [3] reported by Batchelor [4]. The open lozenges are deduced from a compilation of the experimental results obtained with the tracer method - thin film $\mathrm{A}$ on a substrate $\mathrm{B}$ with $\mathrm{B}=\mathrm{Si}, \mathrm{Cu}, \mathrm{Ag}, \mathrm{W}$ - and primary beam energy ranging from $15 \mathrm{keV}$ to $30 \mathrm{keV}$ - see table I in reference [15] in which the fluorescence corrections have been taken into account. 


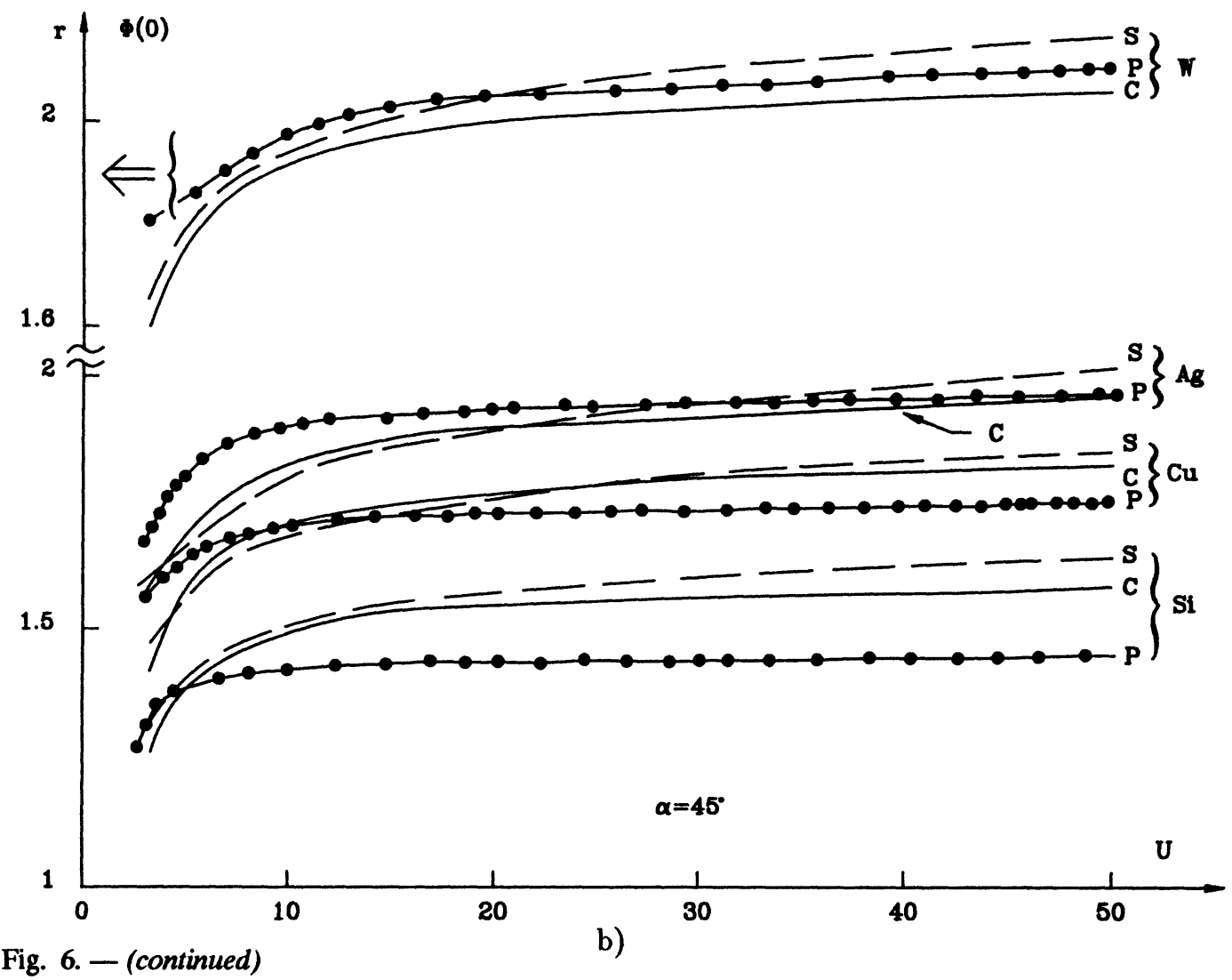

Fig. 6. - (continued)

In fact, the obvious limitation of the proposed expression concerns its use at very low reduced energies $\left(1<U_{0}<2 /(1+\eta)\right)$ where $r$ became negative. Looking at the comparisons shown in figure 6, it seems realistic to suggest its use for $U_{0} \geq 3$ even if the results obtained at $E_{\mathrm{o}} \simeq 5 \mathrm{keV}$ for $\mathrm{Si}(\mathrm{K})$ with $U_{\mathrm{o}} \simeq 2.7$ are not so bad (see Fig. 5a).

\section{Discussion and consequences.}

4.1 Discussion. - Despite the very crude assumptions on which equation (8) has been established, it is surprising to see that it leads to satisfying results compared to more sophisticated methods.

We believe that its success is based on the fact that it only takes into account the physical parameters influencing $r$, or $\phi(0)$, i.e. mainly the backscattering coefficient $\eta$ which is directly responsible of the ionization of the surface atoms while most of the other approaches (such as that of Shimizu) are focussed on the indirect cause of these ionizations, the substrate composition.

In the Shimizu and Pouchou approaches, the need to find functional expressions valid for all the elements composing the substrates induces also a loss of accuracy in the final results.

For the physical point of view, the advantage of the proposed approach, focussed on $\eta$, is to understand easily the general behaviour of $r$ and its boundary conditions.

i) When $\eta$ zero, $R$ is obviously null 
ii) When $E_{\mathrm{o}}$ is increased above $10 \mathrm{keV} Q(\bar{E}) / Q\left(E_{\mathrm{o}}\right)$ approaches unity while changes in $\eta$ are very small explaining the asymptotic behaviour of $r$ at high beam energies

iii) As explained in section 3.1, the angular change of $r$ is the result of a competition between the increase of $\eta$ (with $\alpha$ ) and the influence of the weighting factor $\cos \alpha$. This competition explains the specific shape of the silicon curve (Figs. 3a and 4) relative to the always decreasing shape observed on heavier elements (higher $\eta$ values). Consequently the shape of $r(\alpha)$ observed for silicon is expected to be more pronounced for lighter elements $\mathrm{Al}, \mathrm{C}$ and $\mathrm{Be}$ where $\eta$ is less than 0.2. This expected shape is illustrated in figure 7 for Be where, in opposition, the case of gold is also shown.

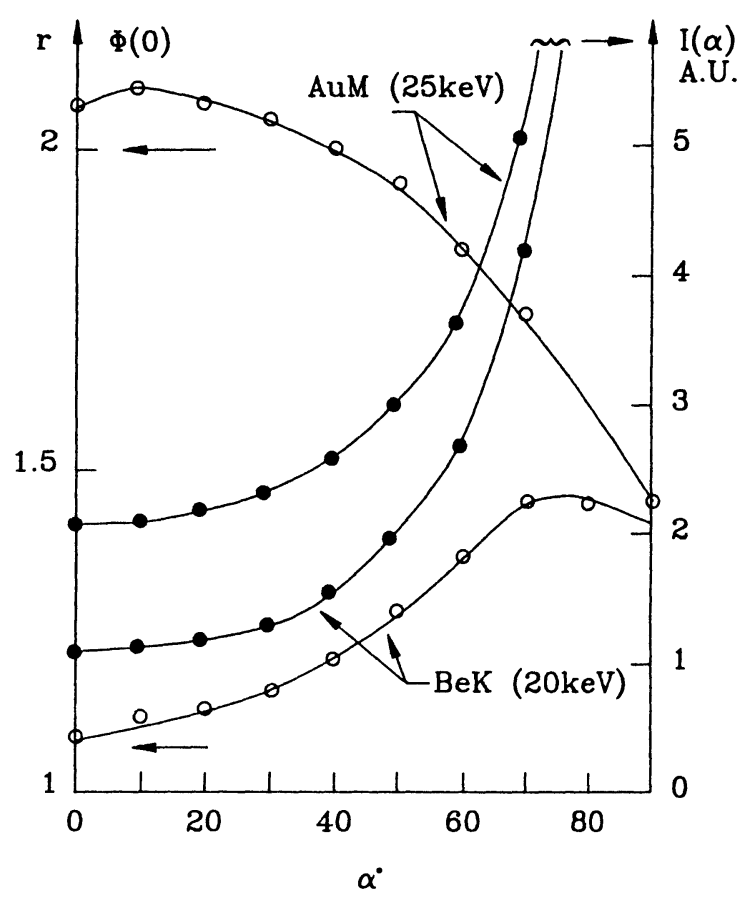

Fig. 7. - Full lines with open symbols (left scale): Auger backscattering correction factor (and $\phi(0)$ function) as a function of the angle of incidence $\alpha$. Calculations from equation (8) using the experimental results of Dresher [23-24] for $\eta(\alpha)$ of two extreme elements (Be: $Z=4$ and Au: $Z=79$ ). Full lines with full symbols (right scale): Auger (or X-ray) intensities emitted by (Be and $\mathrm{Au}$ ) surface atoms as a function of $\alpha$. These last curves are deduced from the formers by dividing $r(\alpha)$ or $\phi_{\alpha}(0)$ by $\cos \alpha$.

4.2 A NEW PROCEDURE FOR PERFORMING THE BACKSCATTERING CORRECTION IN AUGER ANALYSIS. - Over most of the other approaches (except that Love et al. and that of Pouchou et al. for EPMA but only at normal incidence [7]), the proposed expression does not require the knowledge of the substrate composition. This composition is not directly accessible to the Auger experiments while it is the goal of the EPMA experiments. Even known, it leads to complications (and errors) for evaluating $r$ (or $\phi(0)$ ) in complex substrates which are heterogeneous in composition (with complex interfaces between the various microphases) in the thickness of complete diffusion. We hope all these difficulties will be overcome in point analysis as well as in moving 
analysis (X-Y maps, depth profiles) of heterogeneous specimens if the backscattering factor, $\eta$, responsible for the ionization of the surface atoms, is measured. A possibility is to use a calibrated backscattered electron detector in a way similar to that developped by Barkshire et al. for Auger [16, but such a detector is not able to collect all these electrons over $2 \pi$ sterad and the corresponding loss of the signal will be dependent on the angle of incidence and the nature of the investigated specimen (see Fig. 2c). A better procedure seems to measure the specimen current, $I_{\mathrm{s}}$, when the specimen holder is polarised at $V_{\mathrm{o}} \simeq+50$ volts in order to suppress the secondary electron emission $\delta$. In this case $I_{\mathrm{s}}$ is $I_{\mathrm{s}}=I_{\mathrm{o}}(1-\eta)$ and $\eta$ is easily deduced when the incident beam current $I_{\mathrm{o}}$ is measured with a Faraday cup. This procedure has been used by Bishop for the accurate measurement of $\eta[17]$ and we suggest to use it in parallel with the Auger (or the EPMA) analysis of the specimen. Of course this procedure will induce a whole shift of the Auger lines (of $\sim 50 \mathrm{eV}$ ) towards the low kinetic energy range but this effect can be easily taken into account.

In the non-destructive characterization and $x-y$ profilometry of thin coatings by EPMA as initiated recently in our laboratory [13], the proposed measurement of the Auger backscattering factor for the element $A$ on a substrate $S_{i}$ of the investigated specimen, $r\left(\mathrm{~A} / \mathrm{S}_{\mathrm{i}}\right)$, and $r\left(\mathrm{~A} / \mathrm{S}_{\mathrm{r}}\right)$ for the reference specimen: (the same element $A$ of known thickness on a substrate having another composition $S_{r}$ ) will lead to reduce the number of reference specimens to be used for the calibration, (see Eq. (8) in Ref. [13]).

4.3 OTHER REMARKS. - The proposed expression allows the estimation of the influence of the roughness of the surface on the $r$ (or $\phi(0)$ ) values through the local change of the incident angle $\alpha$ associated to this roughness. This influence can be studied analytically from equation (8) if the change of $\eta$ as a function of $\alpha$ is taken into account (Eq. (9) for instance), as well as the change (like $1 / \cos \alpha$ ) of the incident beam contribution on the measured intensities.

From the definitions of $r$ and $\phi(0)$, it is clear that the characteristic intensities emitted by the surface atoms are proportional to $r / \cos \alpha$. (or $\phi(0) / \cos \alpha)$. Thus, dividing the calculated values of $r$ (Figs. 3 and 4) by $\cos \alpha$ allows the estimate of the influence of a local change of $\alpha$ of flat but inclined regions (due to the local roughness or to the tilting of the specimen) on these generated intensities. As shown in figure 7 as an example, it can be deduced that the topographic effects are minimized at normal incidence where a local change of $\Delta \alpha= \pm 20^{\circ}$ does not induce significant changes on the emitted surface intensities for almost all the elements.

This analysis does not mean that the measured intensities do not change and the other factors involved in the Auger or X-ray measurements have to be taken into account. They are the edge effects on non-flat regions (reducing or increasing the number of ionizations relative to the flat surface situation) [20] and the local change of the take-off angles (leading to the change in the attenuation of the detected particles) as well as the shadow effects (the two last effects being correlated to the detector position).

Restricted here to the influence of a local change of the angle of incidence only on the generated intensities, one may also observe that this influence is expected to be maximum close to the grazing incidence (see Fig. 7, the rapid change of $I$ as a function of $\alpha$ in the range $\alpha \simeq 70^{\circ}-90^{\circ}$ ). But in this angular range, the Auger backscattering factor approaches the same numerical value for almost all the elements $(r \simeq 1.4-1.5$ when $\eta \simeq 1$ and $\cos \alpha \simeq 0$ in Eq. (8)). Consequently the advantage of this situation is that the matrix correction factor $r$ of Auger can be neglected.

\section{Conclusion.}

Based on physical arguments, an analytical expression for the Auger backscattering factor and the $\phi(0)$ function of EPMA has been proposed. The results deduced from this expression are in 
good agreement with previous calculations. The advantages of this expression are that it covers the whole angular range of the angles of incidence in an analytical form and that its use does not require the knowledge of the substrate composition.

An experimental procedure for evaluating $r$ (and $\phi(0)$ ) during the Auger and EPMA experiments has been suggested and some influences of the topographic effects have been deduced.

\section{Acknowledgements.}

It is a pleasure to acknowledge C. Merlet (USTL, Montpellier, France), for the critical reading of the manuscript and comments; H. Benhayoune and O. Jbara (LASSI, Reims, France) for their comments and discussion on the experimental aspects.

\section{References}

[1] JABLONSKI A., Surf. Interf. Anal 1 (1979) 122.

[2] BISHOP M.E. and RIVIERE J.C., J. Appl Phys. 40 (1969) 1740.

[3] El Gomati M.M., Broomfield J.P., Matthew J.A.D. and Prutton M., Scanning Electron Microscopy, SEM Inc AMF O'Hare, Chicago, Vol. III (1983) 1101.

[4] BATCHELOR R.D., REZ P., FATHERS D.J. and Venables J.A., Surf. Interf. Anal. 13 (1988) 193.

[5] ICHIMURA S., SHIMIZU R. and LANGERON J.P., Surf. Sci. 124 (1983) 149.

[6] SHIMIZU R., Jpn J. Appl. Phys. 22 (1983) 1631.

[7] LOVE G., COX M.G. and SCOTT V.D., J. Phys. D. Appl Phys. 11 (1978) 23.

[8] PACKWOOD R.H. and BROWN J.D., X-Ray Spectrom. 10 (1981) 138.

[9] TIRIRA SAA J.H. and RIVEROS J.A., X-Ray Spectrom. 16 (1987) 27.

[10] MERLET C., X-Ray Spectrometry, to appear.

[11] POUCHOU J.L.,PICHOIR F. and BOIVIN D., Proceedings of the 12th Intern. Cong. on X ray Optics and Microanalysis, S. Jasienska and L.J. Maksymovicz Eds., Vol. I (1989) 52.

[12] Castaing R., Thèse Univ. Paris (1951); Advances in Electronics and Electron Physics, C. Marton Ed. (Plenum Press, NY) 13 (1960) 317.

[13] CaZauX J., Jbara O., ThOMas X., Surf. Interf. Anal 15 (1990) 567.

[14] CaZAUX J., Mikrochemica Acta B9 (1992) 107

[15] Merlet C., Jbara O., Rondot S. and CazauX J., Proceed. of the ECASIA 5, to appear in Surf. Interf. Anal. 19 (1992).

[16 BARKShIRE R., PRUTTON M., SKInNER D.K., Surf. Interf. Anal. 17 (1991) 213.

[17] BISHOP H.E., in X-Ray optics and Microanalysis, 4th Int. Conf. of X-Ray Optics and Microanalysis, R. Castaing, P. Deschamps and J. Philibert Eds. (1965) p. 153.

[18] SEILER M., Scanning Electron Microscopy, SEM Inc. AMF O’Hare Chicago, Vol. I (1976) 9.

[19] REIMER L., in Scanning Electron Microscopy, Springer Series Opt. Sci. (Springer Verlag Heidelberg) Vol. 45 (1985).

[20] HUNGER H.J. and KUCHLER L., Physica Status Solidi a 56 (1979) K 45.

[21] DARLINGTON E.H., J. Phys. D: Appl. Phys. 8 (1975) 85.

[22] NIEDRIG H., J. Appl. Phys. 53 (1982) R 15.

[23] DRescher H., Reimer L. and Seidel H., Z. Angew. Phys. 29 (1970) 31.

[24] KanaYa K. and Ono S., J. Phys. D: Appl Phys. 11 (1978) 1495.

[25] ARNAL F., VERDIER P. and VINCENSINI P.D., C.R. Acad. Sci. 268 (1969) 1526.

[26 BATCHELOR D.R., D. Phil. Thesis, Univesity of Sussex UK (1985).

[27] JABLONSKI A., Surf. Interf. Anal 2 (1980) 39.

[28] JBARA O., Thesis, University of Reims F (1991) unpublished.

[29] El Gomati M.M., Prutton M., Lamb B. and TupPen C.G., Surf. Interf. Anal 11 (1988) 251.

[30] Cazaux J., Bardoux T., Mouze D., Patat J.M., Salace G., Thomas X., Toth J., Proc. of the ECASIA 5, to appear in Surf. Interf. Anal 19 (1992). 